\title{
Multi-TeV CLIC Photon Collider Option
}

\author{
H. Burkhardt
}

Considerations for an option of $\gamma \gamma$ collisions at multi-TeV energies are described, with focus on the $3 \mathrm{TeV}$ CLIC machine, currently under study at CERN. 


\title{
Multi-TeV CLIC Photon Collider Option
}

\author{
H.Burkhardt
}

CERN, Geneva, Switzerland

\begin{abstract}
Considerations for an option of $\gamma \gamma$ collisions at multi- $\mathrm{TeV}$ energies are described, with focus on the $3 \mathrm{TeV}$ CLIC machine, currently under study at CERN.
\end{abstract}

Key words: Linear accelerators, Compton scattering, Lasers

PACS: 29.27.-a, 13.60.Fz, 42.55.-f

\section{Introduction, Motivation}

In general, any single pass linear $\mathrm{e}^{+} \mathrm{e}^{-}$collider can also become a $\gamma \gamma$, $\gamma e$ or $\mathrm{e}^{-} \mathrm{e}^{-}$collider. For the photon options, it is in principle sufficient to foresee the possibility of pointing an intense low energy photon beam (typically circularly polarized laser light, roughly at visible wavelength) towards the charged particle beam, such that the photons collide with the $\mathrm{e}^{+}$or $\mathrm{e}^{-}$particles just upstream of the IP. The large cross section and the kinematics of the Compton scattering process result in a rather efficient transfer of the electron energy and polarization to the photons. The photon option can be considered as a natural, and potentially cheap extension of the physics capabilities of the linear collider.

Now, do these general considerations remain valid at very high energy?

One can argue, that multi $\mathrm{TeV}$ colliders will be rather big and expensive machines with significant backgrounds and broadened energy spectra due to beamstrahlung. The heavy investment needed to design and built a multi TeV collider should be fully exploited and the addition of the laser interactions may be an affordable extension. Furthermore, there is no beamstrahlung in pure $\gamma \gamma$ and $\gamma e$ collisions (beamstrahlung is synchrotron radiation, produced by charged particles of one beam in crossing the electromagnetic fields of the opposing charged beam). It appears therefore to be well justified to look more closely into the photon option for multi TeV colliders. 


\section{CERN planning, CLIC}

The $\mathrm{e}^{+} \mathrm{e}^{-}$colliding beam storage ring LEP at CERN currently runs with excellent performance, well beyond design at centre-of-mass energies exceeding $208 \mathrm{GeV}$. LEP is however scheduled to stop soon to allow for installation of the $7+7 \mathrm{TeV}$ proton-proton collider LHC. The LHC project requires almost all CERN resources up to year 2008.

Based on past experience, the planning and construction of future high energy machines is expected be a long (difficult and expensive) process. LEP planning and construction spanned a period of 16 years from 1973 to 1989 and there will be about 20 years from 1985 to 2005 for the LHC. Post LHC studies have therefore already started (using a small percentage of the CERN resources). Three principal future options where identified in a report by J. Ellis, E. Keil and G. Rolandi in 1998 [1]. In order of maturity and current effort these options are:

- linear $\mathrm{e}^{+} \mathrm{e}^{-}$collider with $E_{\text {cms }}>2 \mathrm{TeV}$, CLIC

- multi-TeV $\mu^{+} \mu^{-}$collider, (now first a $50 \mathrm{GeV} \mu$ storage ring $\nu$-factory)

- very large hadron pp ( and ep) collider

The possible design of an $\mathrm{e}^{+} \mathrm{e}^{-}$collider CLIC with a nominal centre-of-mass energy of $3 \mathrm{TeV}$ is currently under study at CERN[2]. A schematic view of the

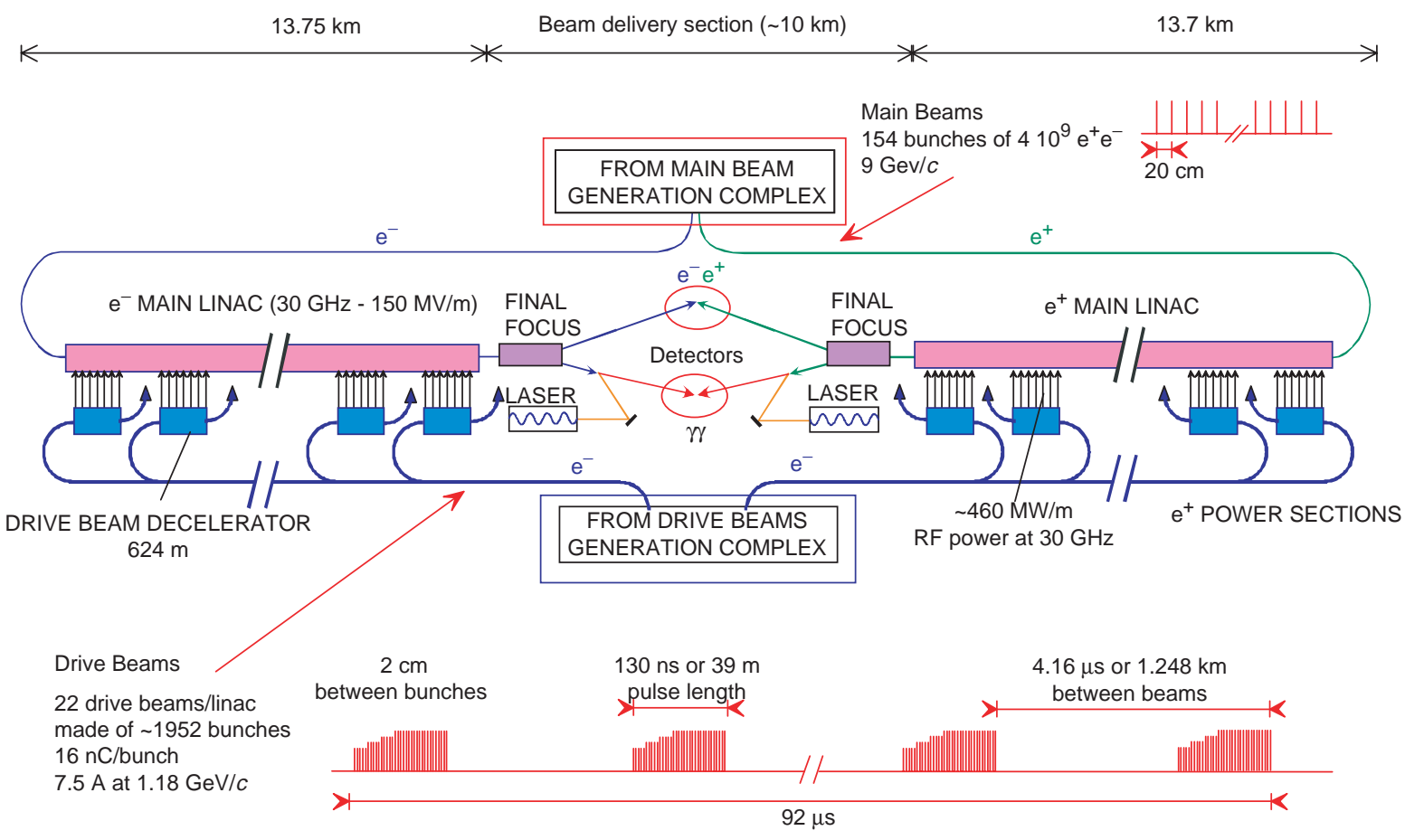

Fig. 1. Overall layout of CLIC for a centre-of-mass energy of $3 \mathrm{TeV}$. 
overall layout is shown in Fig. 1. The electrons and positrons are accelerated in a single pass in the two main linacs and brought into collision in one or two interaction regions. The design is based on two-beam acceleration at a frequency of $30 \mathrm{GHz}$. The nominal gradient for $3 \mathrm{TeV}$ operation is $150 \mathrm{MV} / \mathrm{m}$.

\section{Compton scattering}

The process of electron photon or Compton scattering is well known and described in standard textbooks. We will use here the formulas and notation of Ginzburg et al. [3,4], well suited for $\gamma \gamma$ collisions in linear colliders.

Compton scattering played a role in different contexts in LEP. Circularly polarized laser light, back-scattered off the electrons, is used for energy calibration purposes in LEP to monitor transverse polarization of the beam particles [5]. Compton scattering is also a source of unwanted background particles. Photons from synchrotron radiation, scattered off electrons in the beam pipe or collimators, can reach the experiments. The scattering of beam particles and thermal photons emitted from the beam pipe by black body radiation reduces the beam lifetime [6-8].

The kinematics of the Compton scattering process as seen in the lab frame is shown in Fig. 2.

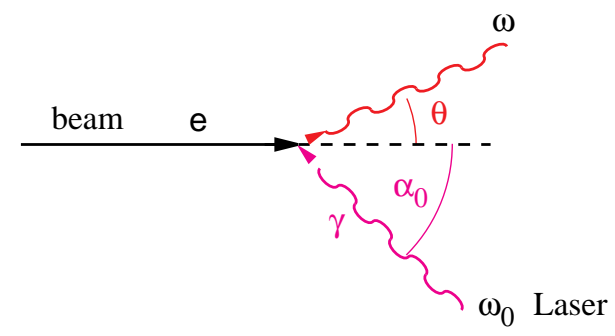

Fig. 2. Kinematics of Compton scattering in the lab frame.

The maximum energy $\omega_{m}$ of the scattered photons is

$$
\omega_{m}=\frac{x}{x+1} E_{b}
$$

$x$ is the momentum transfer squared, normalized to the electron energy $E_{b}$

$$
x=\frac{4 E_{b} \omega_{0} \cos ^{2} \alpha / 2}{m^{2} c^{4}} \simeq 15.3\left[\frac{E_{b}}{\mathrm{TeV}}\right]\left[\frac{\omega_{0}}{\mathrm{eV}}\right]=19\left[\frac{E_{b}}{\mathrm{TeV}}\right]\left[\frac{\mu \mathrm{m}}{\lambda}\right]
$$

Fig. 3 shows the scattered photon spectrum for several beam energies and electron and laser photon helicities (positive and negative helicity product as 
dashed curves and the solid lines for the unpolarized case), at a fixed initial laser photon energy of $\omega_{0}=1 \mathrm{eV}$. At high energies, the photon spectrum

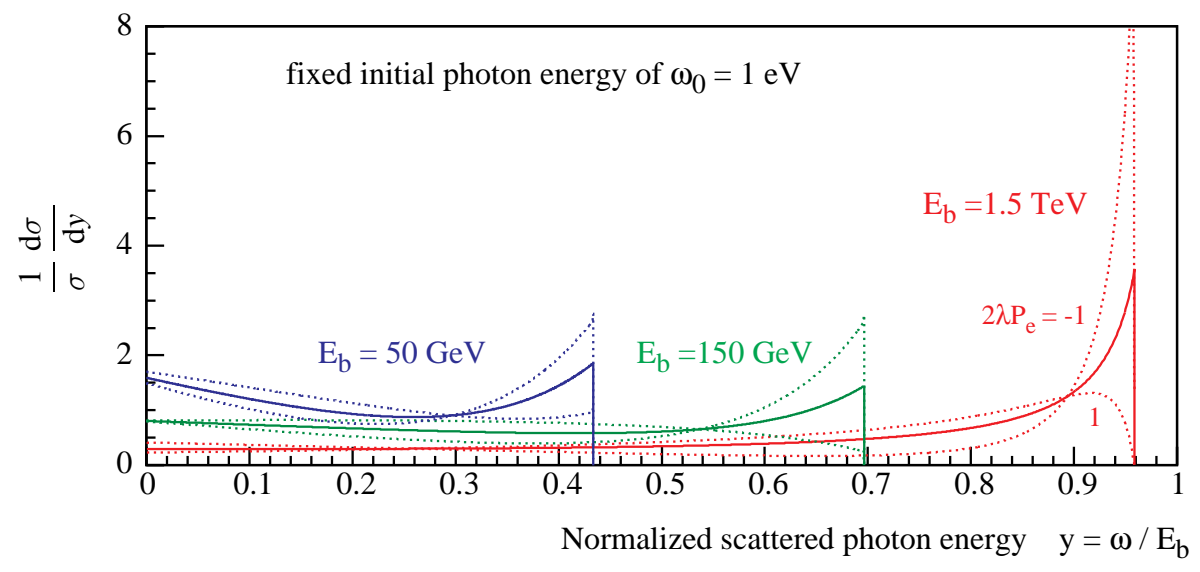

Fig. 3. Scattered photon energy spectrum.

becomes rather peaked towards the beam energy. This is further enhanced for longitudinally polarized electrons and photons in the case of a negative product of helicities.

Cross sections and kinematics are identical for $\mathrm{e}^{+} \gamma$ and $\mathrm{e}^{-} \gamma$ interactions. In practice, it will be advantageous to obtain $\gamma \gamma$ collisions from $\mathrm{e}^{-} \mathrm{e}^{-}$rather than $\mathrm{e}^{+} \mathrm{e}^{-}$beams. This is due to the availability of polarized electron sources and the reduction in the background electron-electron luminosity through the anti-pinch effect in collisions of equal charges.

\section{Choice of laser wavelength, Background}

The outgoing high energy photons can scatter again with other photons of the laser beam if the energies exceed the threshold for the inverse annihilation (Bethe-Wheeler process $\gamma \gamma \rightarrow \mathrm{e}^{+} \mathrm{e}^{-}$). The threshold is $\omega_{m} \omega_{0}=m_{e}^{2} c^{4}$ or $x=$ $2(1+\sqrt{2}) \approx 4.83$. For a beam energy of $1.5 \mathrm{TeV}$, operation will be above this threshold unless the laser photon energy is chosen in the infrared with wavelength $\lambda>5.9 \mu \mathrm{m}$.

Long laser wavelengths, of order of several $\mu \mathrm{m}$ are expected to be difficult in several respects:

- availability of high power, short pulse lasers

- laser spot size (diffraction limit)

- nonlinear QED

The best compromise between signal and background should be determined by detailed simulation and may well be somewhat above $x=4.83$ [9]. Anyway, 
even below $\mathrm{x}=4.83$, one will always have to deal with a mixture of $\gamma \gamma, e \gamma$ and $e e$ collisions, rather broad luminosity-energy spectra, and secondary interactions involving photons from beamstrahlung. This can be seen in Fig. 4 (prepared for TESLA, $\left.E_{\mathrm{cms}}=0.5 \mathrm{TeV}[10]\right)$.

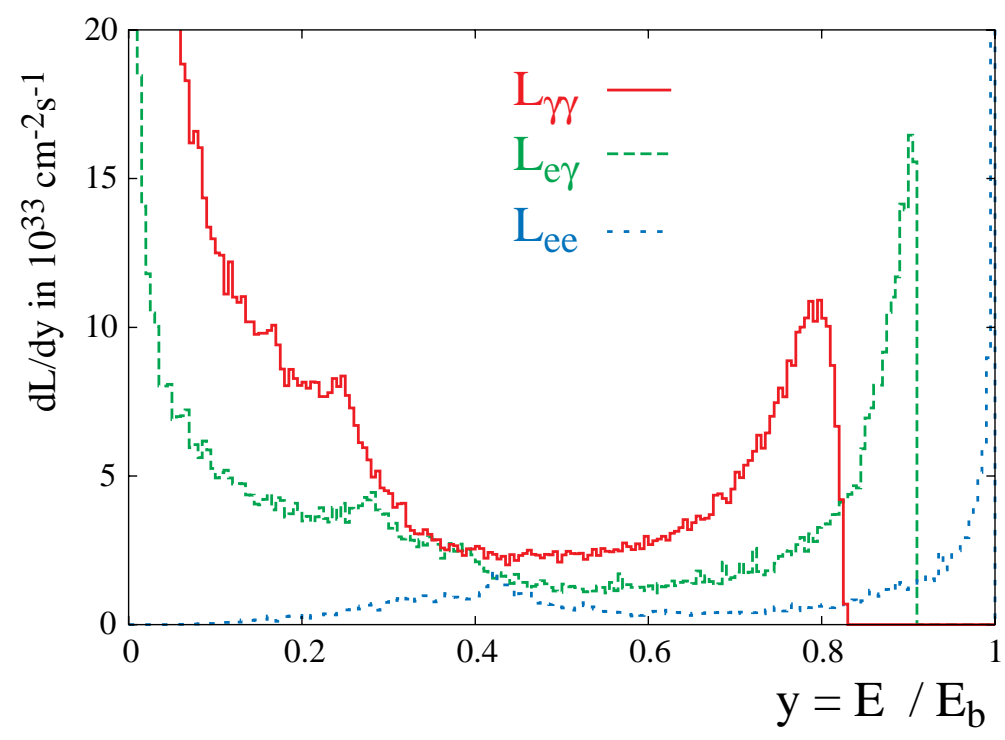

Fig. 4. Typical luminosity spectra in $\gamma \gamma$ collisions.

A very schematic view of a possible layout of the interaction region in CLIC with $\gamma \gamma$ collisions is shown in Fig. 5 . The beams cross at an angle of $\pm 10 \mathrm{mrad}$.

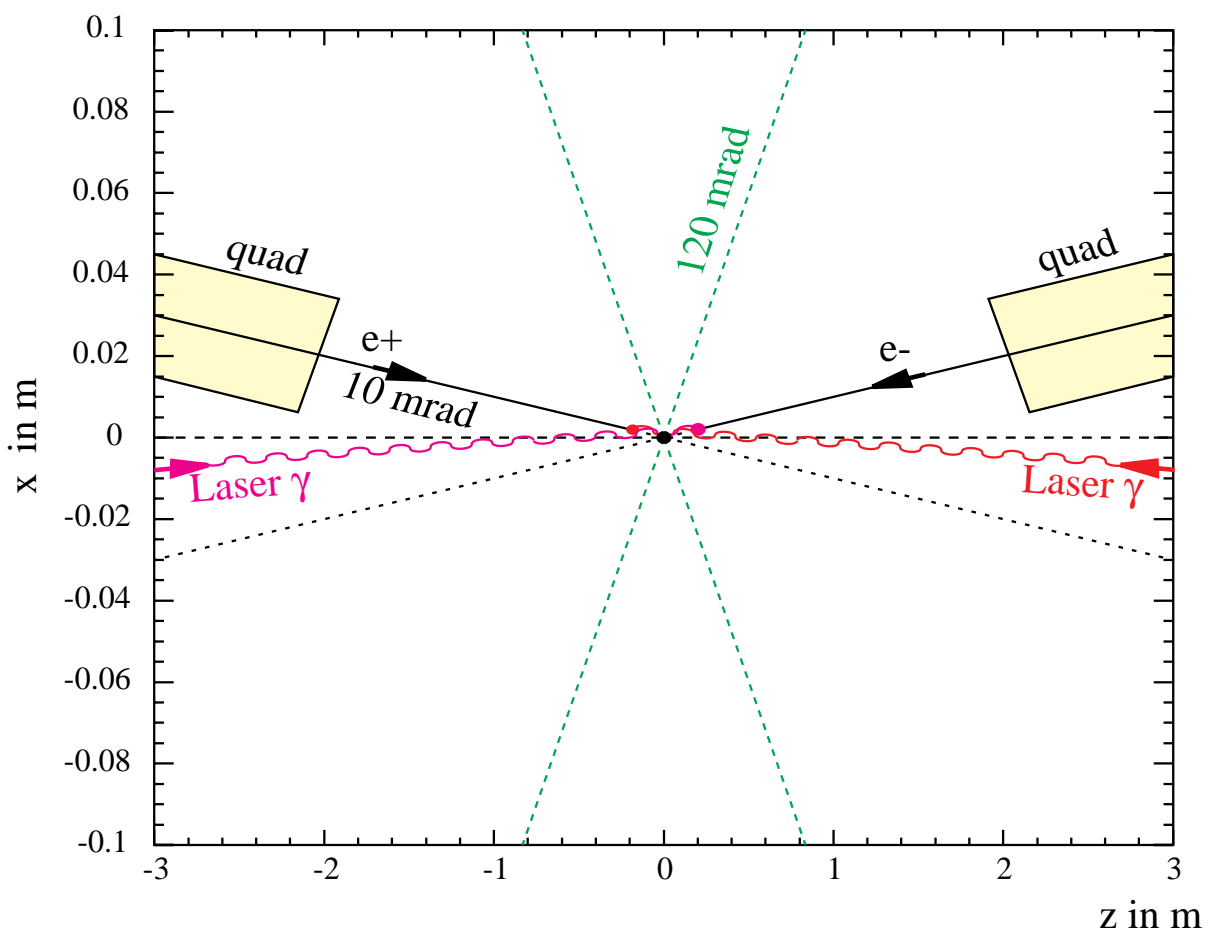

Fig. 5. Schematic interaction region layout with $\gamma \gamma$ collisions 
The $\gamma \gamma$ interaction spot size should be very small, of order $\sigma_{y}=1 \mathrm{~nm}$ to achieve a high luminosity. This implies, that the laser - electron interaction points will have to be very close to the $\gamma \gamma$ interaction point (IP), at a distance of roughly $b=\gamma \sigma_{y}=3 \mathrm{~mm}$.

The current working optics version for a $3 \mathrm{TeV}$ CLIC foresees a beam delivery system (BDS) of about $3.3 \mathrm{~km}$ length with a single interaction point (IP) [11]. For the $\gamma \gamma$ option, one usually assumes a second, independent IP equipped with lasers and mirrors and beam optics optimized for $\gamma \gamma$ interactions. This would be rather difficult and expensive at $3 \mathrm{TeV}$ and imply a duplication of the BDS. For the present CLIC study, the priority is on a design with a single IP, possibly equipped with two movable detectors.

Fig. 6 shows how a solution with two IP's might look like. The average horizontal crossing angle of $\pm 10 \mathrm{mrad}$ is obtained by construction of the two linacs.

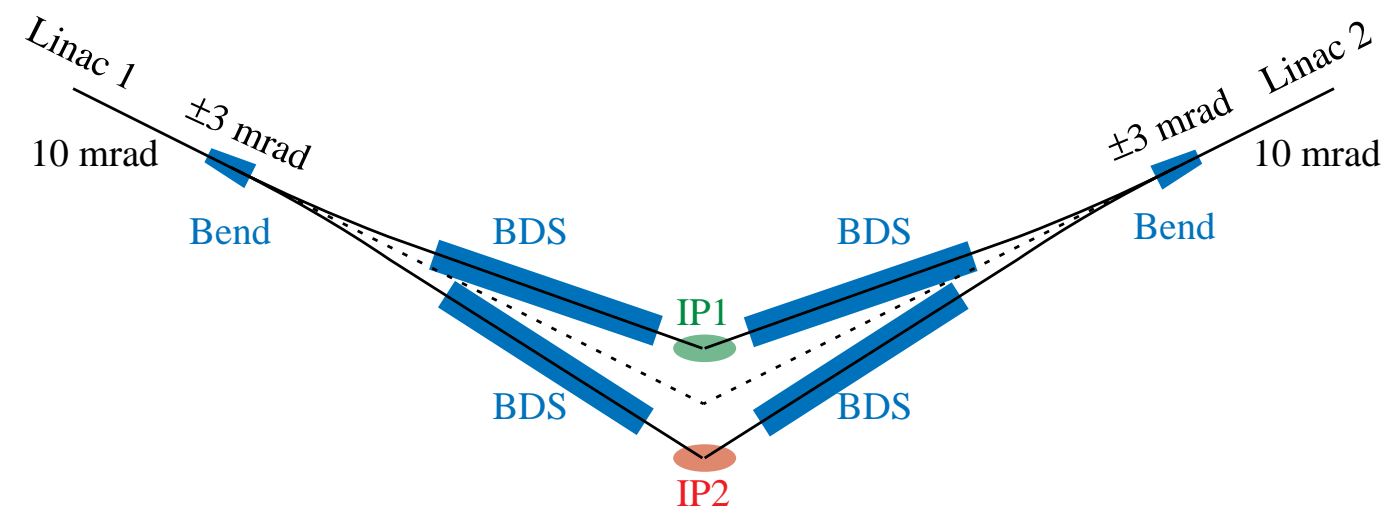

Fig. 6. Possible layout with two interaction regions. Switching between the IP's is achieved by a change of polarity in the bends.

A separation of $24 \mathrm{~m}$ between the two interaction points could be achieved with bending angles of $\theta= \pm 3 \mathrm{mrad}$ at a distance of $4 \mathrm{~km}$. The synchrotron radiation loss in the extra bends can be estimated as follows. For a field of $0.05 \mathrm{~T}$ (corresponding to a bending radius of $\rho=100 \mathrm{~km}$ ), the length of the bending magnet is $L=300 \mathrm{~m}$. At $1.5 \mathrm{TeV}$, the average number of photons radiated over this length is ( $\alpha$ is the fine structure constant, $\gamma$ the Lorentz factor)

$$
N_{\gamma}=\frac{5 \alpha \gamma L}{2 \sqrt{3} \rho}=\frac{5 \alpha \gamma \theta}{2 \sqrt{3}}=92.8
$$

and the average photon energy

$$
E_{\gamma}=\frac{8}{15 \sqrt{3}} E_{\text {crit }}=23 \mathrm{MeV}
$$


implying an energy loss of $1.4 \times 10^{-3}$ and an energy spread of $3 \times 10^{-4}$, which seems tolerable.

\section{Conclusions}

A $\gamma \gamma$ option for a multi-TeV linear collider like the $3 \mathrm{TeV}$ CLIC, is attractive and should be studied in detail. Some first, preliminary considerations have been presented here. A challenge at high energies is to find an acceptable com-

promise between an increase in laser wavelength and additional background from multiple Compton interactions.

\section{References}

[1] J. Ellis, E. Keil, and G. Rolandi, "Options for Future Colliders at CERN,". CERN-EP-98-03.

[2] The CLIC study team, Ed. G. Guignard, "A 3 TeV e ${ }^{+} \mathrm{e}^{-}$Linear Collider Based on CLIC Technology." CERN yellow report CERN 2000-008, July 2000.

[3] I. F. Ginzburg, G. L. Kotkin, V. G. Serbo, and V. I. Telnov, "Colliding $\gamma e$ and $\gamma \gamma$ beams based on the single pass $\mathrm{e}^{+} \mathrm{e}^{-}$accelerators (VLEPP type)," Nucl. Instr. Meth. 205 (1983) 47.

[4] I. F. Ginzburg, G. L. Kotkin, S. L. Panfil, V. G. Serbo, and V. I. Telnov, "Colliding $\gamma \mathrm{e}$ and $\gamma \gamma$ beams based on the single pass $\mathrm{e}^{+} \mathrm{e}^{-}$accelerators. 2. Polarization effects. Monochromatization improvement," Nucl. Instr. Meth. A219 (1984) 5-24.

[5] M. Placidi and R. Rossmanith, " $\mathrm{e}^{+} \mathrm{e}^{-}$Polarimetry at LEP," Nucl. Instrum. Meth. A274 (1989) 79.

[6] G. von Holtey, A. Ball, et al., "Study of beam-induced particle backgrounds at the LEP detectors," Nucl. Instrum. Meth. A403 (1998) 205-246.

[7] B. Dehning, A. C. Melissinos, F. Perrone, C. Rizzo, and G. von Holtey, "Scattering of high-energy electrons off thermal photons," Phys. Lett. B249 (1990) 145-148.

[8] H. Burkhardt, "Monte Carlo Simulation of Beam Particles and Thermal Photons," SL Note 93-73 (OP), CERN, July, 1993.

[9] V. Telnov, "Problems with multi-TeV photon colliders." these proceedings.

[10] D. Schulte, "Study of electromagnetic and hadronic background in the interaction region of the TESLA Collider,". DESY-TESLA-97-08. 
[11] F. Zimmermann, R. Assmann, G. Guignard, D. Schulte, and O. Napoly, "FinalFocus System for CLIC at 3 TeV." CERN-SL-2000-057, CLIC Note 446, (proc. EPAC 2000). 